\title{
The cranial-side parietal pleura pick-up method and the edge closure technique as a closure method for open thoracotomy
}

\author{
Noriaki Sakakura, Hiroaki Kuroda \\ Department of Thoracic Surgery, Aichi Cancer Center Hospital, Nagoya, Japan \\ Correspondence to: Noriaki Sakakura, MD, PhD. Department of Thoracic Surgery, Aichi Cancer Center Hospital, 1-1 Kanokoden, Chikusa-ku, \\ Nagoya 464-8681, Japan. Email: nsakakura@aichi-cc.jp.
}

Submitted May 08, 2020. Accepted for publication Jul 01, 2020.

doi: $10.21037 /$ jtd-20-1212

View this article at: http://dx.doi.org/10.21037/jtd-20-1212

Given the delicate intercostal maneuvers required during closure for basic open thoracotomies, two methods, both involving the caudal side of the thoracotomy, have been reported; namely suturing through small holes drilled in the caudal rib (intracostal suture) (1) and suturing the thin space between the inferior edge of the caudal rib and the intercostal neurovascular bundle to prevent strangulation of the intercostal nerve and vessels on the caudal side (edge closure technique) $(2,3)$.

Apart from the aforementioned methods, a closure method that involves picking up the parietal pleura attached to the intercostal muscle on the cranial side of the thoracotomy using sutures has been utilized. This method has been used by a small number of surgeons, without any suitable name or relevant reports. Furthermore, the intrathoracic chest wall condition after utilizing this method has not been confirmed. We have now called this procedure the "cranial-side parietal pleura pick-up method" in English and Tosoku kyomaku sukuiage-ho in Japanese and have routinely used this method in combination with the aforementioned pain-reducing procedures. We herein describe this delicate procedure using comprehensive illustrations and an accompanying supplemental video, and briefly discuss relevant thoracic closure procedures, such as the edge closure technique in the hope of assisting general thoracic surgeons, particularly young trainees and residents, in advancing their clinical skills.

We have regularly adopted either the vertical muscle sparing or splitting thoracotomy (VMST) or alternative VMST procedure for routine open thoracotomies after carefully choosing the type of skin incision that affords the optimum surgical field (4). "Sparing" of the serratus anterior muscle is usually utilized in thoracotomies involving the fifth or more caudal intercostal spaces, whereas "splitting" is applied to procedures involving the fourth or more cranial intercostal spaces. After exposing the bony thorax, the chest is opened conventionally by dissecting the intercostal muscle at the top of the caudal rib using electrocautery. To expand the thoracotomy, a silicon wound retractor (Alexis Wound Protector/Retractor, M; Applied Medical, Rancho Santa Margarita, CA, USA) and two minimally invasive thoracic openers were used.

During closure, sutures were placed around the caudal rib using either the small-hole drilling method (Figure 1A) or edge closure technique (Figure 1B), depending on the surgeon's discretion. Subsequently, the cranial ribs were sutured from the inside to the outside of the thorax, and part of the soft tissue of the cranial intercostal muscle and surrounding parietal pleura were picked up and lifted with the sutures (cranial-side parietal pleura pick-up method; Figure 1C). Routinely, the chest was closed using four to five large blunt needles threaded with No. 1 polydioxanone sutures (PDS Plus, 48 mm, 1/2c; Ethicon, Inc., Somerville, NJ, USA), which were tied while reducing tension by pulling the ribs closer using other sutures. Video 1 shows the closure of a thorax using the edge closure technique and the cranial-side parietal pleura pick-up method, which was visualized using a thoracoscope inserted through a port created for the thoracic chest tube. The photographs in the figure were depicts key scenes from the video with sequential images. The closure without picking up the pleura exposed the dissected portion of the intercostal muscle to the thoracic cavity, with subsequent oozing being observed. In contrast, the pick-up method uniformly 

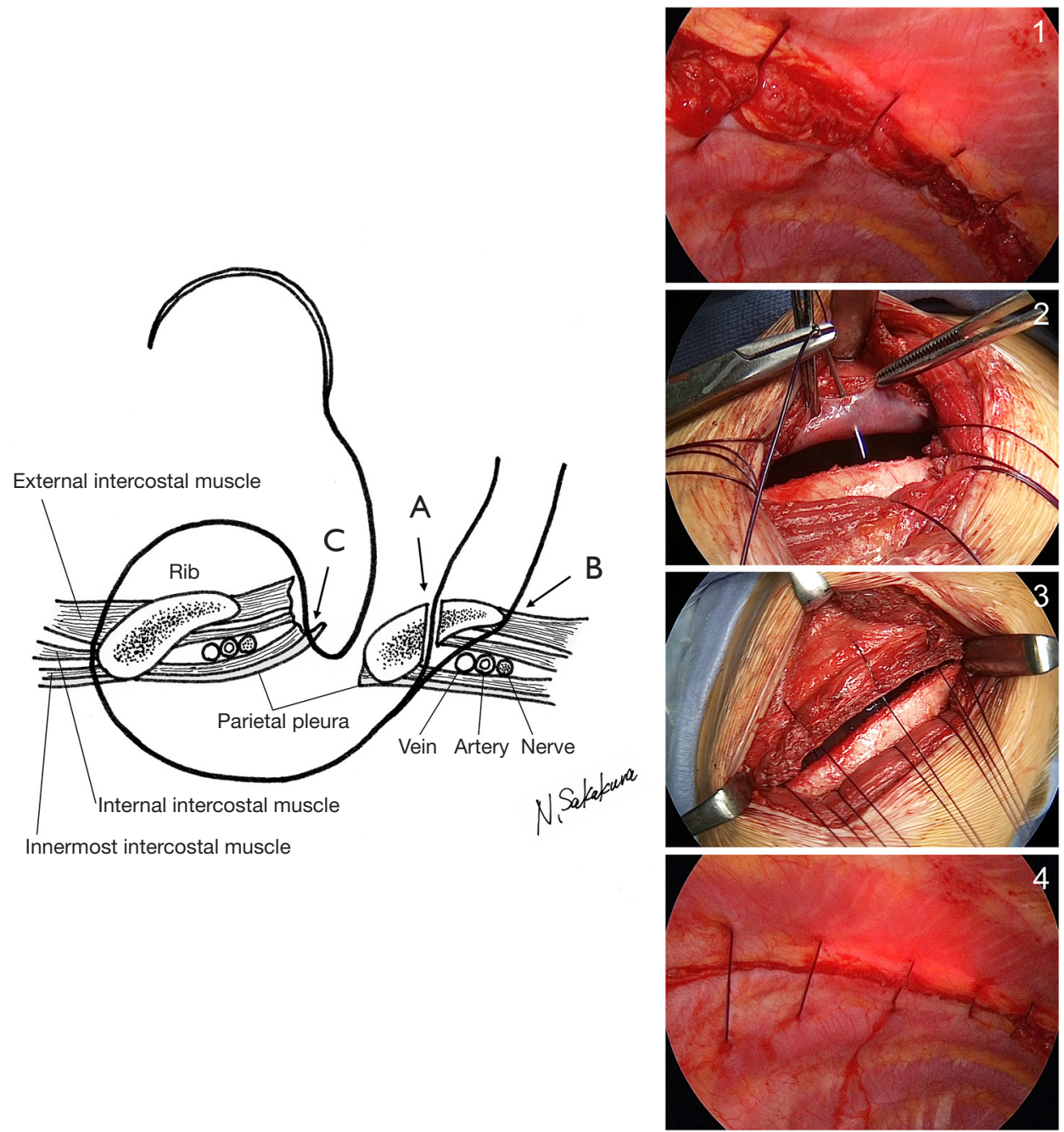

Figure 1 Schema and surgical findings of the procedures. Illustration shows the small-hole drilling method (A), the edge closure technique (B), and the cranial-side parietal pleura pick-up method (C). Photographs are key scenes from Video 1 appearing in sequential order; 1 , thoracic closure without picking up the pleura exposed the dissected portion of the intercostal muscle to the thoracic cavity, with subsequent oozing being observed; 2, the parietal pleura in the cranial side of the thoracotomy was picked up and lifted using the sutures. 3 , the edge closure technique and pick-up method were used in the caudal and cranial sides of the thoracotomy, respectively; 4, after the pick-up method, the dissected portion was uniformly covered by the parietal pleura, with the inner wall at the thoracotomy site being smooth.

covered the dissected portion with the parietal pleura, promoting a smooth chest wall on intrathoracic side.

A survey of thoracic surgeries performed at our institution over the 6-year period from January 2013 to June 2019 revealed that among the 2,434 procedures performed, 1,285 cases required thoracoscopic surgery, while 618 underwent open thoracotomy. Among the latter, 220 cases underwent a procedure utilizing the pick-up method described herein.

To the best of our knowledge, no intrathoracic findings following this procedure have been described previously. While this method may possibly prevent oozing from the intercostal muscle or unexpected hemorrhage from intercostal vessels and reduce adhesions between the lungs and chest wall, quantitative evaluation of such effects may be difficult. Some points to be noted while using this method include the following: (I) the pick-up sutures should be passed carefully given that the parietal pleura is fragile; (II) the sutures should be tied while reducing tension by pulling the ribs closer using other sutures and be pulled 
gently while ensuring that tension is placed toward the direction of the caudal rib; and (III) attention should be paid not to place the sutures too deeply to avoid injury to the intercostal nerves and vessels hidden within the muscle.

The pick-up method itself is a very simple procedure. We emphasize here that a surgical procedure is an accumulation of many small but reliable techniques, the recognition of which may lead to more sophisticated surgeries. We hope that this brief report would prompt further discussion for open thoracotomy, which has recently become less frequent among young thoracic surgery trainees and residents, and contribute to the improvement of their surgical techniques in routine clinical practice.

\section{Acknowledgments}

NS learned the cranial pleura pick-up method from Dr. Tatsuo Uchida. We would like to thank him for his instructions regarding this procedure.

Funding: None.

\section{Footnote}

Provenance and Peer Review: This article was a free submission to the journal. The article did not undergo external peer review.

Conflicts of Interest: Both authors have completed the ICMJE uniform disclosure form (available at http://dx.doi. org/10.21037/jtd-20-1212). The authors have no conflicts of interest to declare.

Cite this article as: Sakakura N, Kuroda H. The cranial-side parietal pleura pick-up method and the edge closure technique as a closure method for open thoracotomy. J Thorac Dis 2020;12(9):5053-5055. doi: 10.21037/jtd-20-1212
Ethical statement: The authors are accountable for all aspects of this work in ensuring that questions related to the accuracy or integrity of any part of the work are appropriately investigated and resolved.

Open Access Statement: This is an Open Access article distributed in accordance with the Creative Commons Attribution-NonCommercial-NoDerivs 4.0 International License (CC BY-NC-ND 4.0), which permits the noncommercial replication and distribution of the article with the strict proviso that no changes or edits are made and the original work is properly cited (including links to both the formal publication through the relevant DOI and the license). See: https://creativecommons.org/licenses/by-nc-nd/4.0/.

\section{References}

1. Cerfolio RJ, Price TN, Bryant AS, et al. Intracostal sutures decrease the pain of thoracotomy. Ann Thorac Surg 2003;76:407-11; discussion 411-2.

2. Sakakura N, Usami N, Taniguchi T, et al. Assessment of long-term postoperative pain in open thoracotomy patients: pain reduction by the edge closure technique. Ann Thorac Surg 2010;89:1064-70.

3. El-Hag-Aly MA, Nashy MR. Double edge closure: a novel technique for reducing post-thoracotomy pain. A randomized control study. Interact Cardiovasc Thorac Surg 2015;21:630-5.

4. Sakakura N, Mizuno T, Arimura T, et al. Design variations in vertical muscle-sparing thoracotomy. J Thorac Dis 2018;10:5115-9. 